\title{
Impact of motor therapy with dynamic body-weight support on Functional Independence Measures in traumatic brain injury: An exploratory study
}

\author{
Emily Anggelis ${ }^{\mathrm{a}}$, Elizabeth Salmon Powell ${ }^{\mathrm{b}}$, Philip M. Westgate ${ }^{\mathrm{c}}$, Amanda C. Glueck $^{\mathrm{d}}$ \\ and Lumy Sawaki ${ }^{\mathrm{b}, *}$ \\ ${ }^{a}$ University of Kentucky, College of Medicine, Lexington, KY, USA \\ ${ }^{\mathrm{b}}$ Department of Physical Medicine and Rehabilitation, University of Kentucky, Lexington, KY, USA \\ ${ }^{\mathrm{c}}$ Department of Biostatistics, University of Kentucky, College of Public Health, Lexington, KY, USA \\ ${ }^{\mathrm{d}}$ Department of Neurology, University of Kentucky, Lexington, KY, USA
}

\begin{abstract}
.
BACKGROUND: Contemporary goals of rehabilitation after traumatic brain injury (TBI) aim to improve cognitive and motor function by applying concepts of neuroplasticity. This can be challenging to carry out in TBI patients with motor, balance, and cognitive impairments.

OBJECTIVE: To determine whether use of dynamic body-weight support (DBWS) would allow safe administration of intensive motor therapy during inpatient rehabilitation and whether its use would yield greater improvement in functional recovery than standard-of-care (SOC) therapy in adults with TBI.

METHODS: Data in this retrospective cohort study was collected from patients with TBI who receive inpatient rehabilitation incorporating DBWS $(n=6)$ and who received inpatient rehabilitation without DBWS (SOC, $n=6)$. The primary outcome measure was the change in Functional Independence Measures (FIM) scores from admission to discharge.

RESULTS: There was significant improvement in total FIM scores at discharge compared to admission for both the DBWS $(p=0.001)$ and SOC $(p=0.005)$ groups. Overall, the DBWS group had greater improvement in total FIM score and FIM subscales compared to the SOC group.

CONCLUSIONS: Our results suggest DBWS has the potential to allow a greater intensity of therapy during inpatient rehabilitation and yield better outcomes compared to SOC in patients with TBI.
\end{abstract}

Keywords: Rehabilitation, inpatient, neuroplasticity, trauma, humans, assistive technology

This material has been presented during the 2019 Association of Academic Physiatrists' Annual Meeting, February 19-23, 2019, Puerto Rico, USA. No reprints are available.

*Address for correspondence: Lumy Sawaki, MD, PhD, University of Kentucky, Department of Physical Medicine and Rehabilitation at Cardinal Hill Hospital, 2050 Versailles Road, Lexington, KY 40504, USA. Tel.: +1 859/323 6226; Fax: +1 859/323 1123; E-mail: 1sawa2@uky.edu.

\section{Introduction}

Traumatic brain injury (TBI) is estimated to affect around 1.7 million people in the United States yearly ("Centers for Disease Control and Prevention. Traumatic Brain Injury \& Concussion,” April 27, 2017). TBI is a serious public health concern due to the fact that those who survive moderate to severe TBI 
commonly have chronic disability and morbidity, including cognitive and motor impairments ("Centers for Disease Control and Prevention. Traumatic Brain Injury \& Concussion," April 27, 2017). Cognitively, TBI has been shown to most commonly affect attention, memory, and executive function (Arciniegas, Held, \& Wagner, 2002). Motor deficits after TBI can be due to several reasons including muscle weakness and spasticity as well as balance and coordination disturbances (Peterson \& Greenwald, 2015). This patient population requires substantial rehabilitation care and TBI related costs contribute to approximately $\$ 76$ million in medical expenses and lost wages in the United States (Corso, Finkelstein, Miller, Fiebelkorn, \& Zaloshnja, 2006). Current standard interventions for TBI include a multidisciplinary approach that involves physicians, nurses, neuropsychologists, occupational therapists, physical therapists, speech/language therapists, and social workers among others to provide patient-specific care (Brasure et al., 2012; Turner-Stokes, Pick, Nair, Disler, \& Wade, 2015).

Contemporary goals of rehabilitation after TBI aim to improve both cognitive and motor function by applying concepts of neuroplasticity (Dobkin, 1993). The idea of neuroplasticity is to re-instate or make adaptive changes to certain neural pathways that once allowed the patient to function independently (Nudo, 2011). Literature as far back as the mid-1900s supports the idea that the injured brain is receptive to neuroplasticity (Glees \& Cole, 1949). A mapping study on non-human primates with focal injury showed adjacent cortical regions of an injured brain adapting to behavioral therapy (Glees \& Cole, 1949) Generally speaking, neuroplastic change occurs more readily as therapy becomes more intensive and repetitive (Kleim \& Jones, 2008; Nudo, 2011). Additionally, such therapy has a greater likelihood of leading to lasting change in functional recovery if the therapy is presented as task-oriented, with progressive challenge, with a requirement of active participation, problem-solving, and attention to task (Kleim \& Jones, 2008; Wolf et al., 2006). Weaver et al. showed in healthy adults that the initial phase of motor skill learning requires activation of cognitive circuits to allow for the attention and planning necessary to perform a movement, demonstrating that cognitive and motor functions are tightly connected (Weaver, 2015). While the concepts of neuroplasticity and task intensity are now widely accepted, it can be challenging to carry it out in inpatient rehabilitation settings, particularly with TBI patients who have motor and cognitive impairments as well as a fear of falling, thus requiring multiple therapists to help assure safety. Therefore, the risk of such therapy during inpatient rehabilitation can be prohibitive and lead to fewer repetitions of tasks resulting in less potential for neuroplastic change.

There is an explosion of rehabilitation technologies attempting to deliver intensive and repetitive therapy while providing the most cost- and functionallyeffective therapy. Body weight support (BWS) therapy is thought to improve function via better strength, posture, and balance as well as reduce the physical burden on therapists allowing for their undivided attention on the patient and their goal. For example, Mao et al. showed that BWS treadmill therapy improves motor function in patients with neurological injuries more than conventional gait therapy (Mao et al., 2015). Most findings of favorable outcomes when applying BWS however, have been primarily after stroke and spinal cord injury (Bland, Zampieri, \& Damiano, 2011; Mao et al., 2015).

A newer set of technologies can provide dynamic BWS (DBWS) where the system applies a constant force while patients are safely practicing gait, activities of daily living, going up or down stairs, and performing balance exercises. The DBWS system can facilitate safe delivery of therapy following the principles of neuroplasticity without requiring multiple therapists. DBWS is almost imperceptible to the patient, and enables the practice of activities with the high repetitions and intensity needed. Therein, allowing the patient to focus their attention on the task, relieved of the fear of falling. However, there is no evidence that DBWS will allow patients with TBI to train at higher intensities and with greater repetitions during acute inpatient rehabilitation, nor if this type of technology will assist in promoting better health outcomes. Thus, the purpose of this study was to determine whether DBWS could be implemented during inpatient rehabilitation and whether its use during therapy would lead to greater functional improvement compared to standard-of-care (SOC) therapy in adults following TBI. We hypothesized motor therapy with DBWS would yield greater functional improvement than SOC therapy in adults following moderate/severe TBI.

\section{Methods}

\subsection{Design}

This study was a retrospective chart review. All procedures were approved by the University of Ken- 
tucky Institutional Review Board, which granted a waiver of informed consent. All activities took place at Cardinal Hill Rehabilitation Hospital which is a free-standing inpatient rehabilitation hospital with a formal partnership with University of Kentucky. The SOC control group (no DBWS) consisted of historical data from before the installation of the DBWS system (ZeroG v3, Aretech LLC, Ashburn, VA). Admission dates for the DBWS group ranged from June 2016 to April 2018, while the SOC group was prior to June 2016. Inclusion criteria were brain injury of traumatic etiology severe enough to require acute inpatient rehabilitation after discharge from the trauma center. In order to ensure that patients were focused on performing motor therapy rather than acclimating to the system, patients in the DBWS group participated in at least two DBWS sessions during their stay in inpatient rehabilitation. Activities performed using DBWS were determined by the physical therapists.

Outcome measures included functional independence measurement (FIM) scores at admission and discharge. Of primary interest was the comparison of mean changes in FIM from admission to discharge between the two groups.

\subsection{Over-ground gait therapy with DBWS}

Physical therapists previously trained to use DBWS administered the therapy sessions in the DBWS group. Therapy using DBWS was included as part of inpatient daily 3 hours of therapy rather than additional therapy, in accordance with current guidelines for therapies in inpatient rehabilitation (Beaulieu et al., 2019). Specific activities in the DBWS and SOC groups were selected according to the clinical judgement of the therapists.

\subsection{Functional Independence Measurement (FIM)}

FIM consists of 18 items divided into motor and cognitive subscales. The motor subscale includes scores of self-care, sphincter control, mobility, and locomotion. The cognitive subscale includes cognition and social cognition. All items are numerically scored from 1 to 7 (complete dependence to complete independence, respectively). Detailed FIM evaluation item scoring is as follows: A score of "1": Total assist needed; "2" = Maximal assist (patient can perform $25 \%$ of the task); " 3 " = Moderate assist (patient can perform 50\% of the task); "4" = Minimal assist (patient can perform $75 \%$ of the task); " $5 "=$ Supervision needed; "6" = Modified independence (patient uses an assistive device); and "7" = Independence. Therefore, the minimal score on Total FIM is 18 (total assistance), and the maximum score is 126 (complete independence).

\subsection{Statistics}

Analyses were conducted in SPSS 24 (IBM, Armonk, NY) and SAS version 9.4 (SAS Institute, Cary, NC). Patient data were grouped by intervention received, DBWS or SOC. Paired samples $t$-tests were used to determine within-group changes from admission to discharge for total FIM, motor FIM subscale, and cognitive FIM subscale. Independent samples $t$-tests were used to determine between-group differences for admission to discharge change in total FIM, motor FIM subscale and its components (self-care, sphincter control, mobility, locomotion), and cognitive FIM subscale and its components (cognition and social cognition). To account for age as a confounding factor, we utilized linear regression modeling with trial arm (primary interest) and age as the predictors of change.

\section{Results}

Twenty four patients with brain injury used DBWS during the period pre-established for this retrospective study. No adverse events were reported for DBWS or SOC patients. Seven patients were excluded due to having a non-traumatic brain injury. Eight patients who used DBWS as part of outpatient therapy were excluded. Three patients were excluded from analyses because DBWS was applied during only one therapy session. Data from 6 patients with TBI who used the DBWS during inpatient rehabilitation and met inclusion criteria was included for analysis (Table 1). Both groups showed significant improvement in total FIM scores at discharge compared to admission for both the DBWS $(p=0.001)$ and SOC ( $p=0.007)$ groups (Fig. 1). Both the DBWS and SOC groups showed significant improvements on motor FIM and cognitive FIM subscales (Fig. 2). The DBWS group showed significantly greater improvement on both the motor $(p=0.008)$ and cognitive subscales $(p=0.021)$ than the SOC group (Fig. 3). In addition, DBWS was found to produce significant improvements in FIM motor components: selfcare, mobility, and locomotion ( $p=0.012, p=0.007$, $p=0.008$, respectively) compared to SOC. For the 
Table 1

Summary of demographic data

\begin{tabular}{|c|c|c|c|c|c|c|c|c|c|c|}
\hline \multicolumn{6}{|c|}{ Dynamic body weight support (DBWS) group } & \multicolumn{5}{|c|}{ Standard of care (SOC) group } \\
\hline & & & \# & & & & & & & \\
\hline Patient & Sex & Etiology & $\begin{array}{c}\text { DBWS } \\
\text { Sessions }\end{array}$ & Age & $\begin{array}{l}\text { Length of } \\
\text { stay (days) }\end{array}$ & Patient & Sex & Etiology & Age & $\begin{array}{l}\text { Length of } \\
\text { stay (days) }\end{array}$ \\
\hline DBWS1 & M & MVA* & 2 & 15 & 23 & SOC1 & M & BHT & 49 & 16 \\
\hline DBWS2 & M & MVA & 2 & 26 & 15 & SOC2 & M & BHT & 20 & 11 \\
\hline DBWS3 & M & MVA & 3 & 45 & 23 & SOC3 & $\mathrm{F}$ & MVA & 20 & 16 \\
\hline DBWS4 & $\mathrm{M}$ & $\mathrm{BHT}^{\dagger}$ & 4 & 48 & 10 & SOC4 & M & MVA & 26 & 54 \\
\hline DBWS5 & $\mathrm{F}$ & MVA & 4 & 47 & 9 & SOC5 & $\mathrm{F}$ & MVA & 25 & 43 \\
\hline DBWS6 & $\mathrm{F}$ & MVA & 4 & 23 & 12 & SOC6 & $\mathrm{F}$ & Fall & 41 & 10 \\
\hline Mean \pm SEM & & & & $34.0 \pm 5.9$ & $15.3 \pm 2.6$ & & & & $30.2 \pm 4.9$ & $25.0 \pm 7.6$ \\
\hline
\end{tabular}

*motor vehicle accident. ${ }^{\dagger}$ blunt head trauma.

cognitive subscale only the social cognition component was significantly higher for the DBWS group compared to the SOC group $(p=0.003)$ (Fig. 3). Based on the mean results from linear regression modeling to account for age, it was determined that for total FIM, the DBWS group improved 38.6 points more than the SOC group $(p=0.005)$. For motor and cognitive FIM, the DBWS group was found to have improved 27.9 and 8.8 points more than the SOC group ( $p=0.007, p=0.006)$, respectively.

\section{Discussion}

The main finding of this study is that DBWS may improve inpatient health outcomes at time of discharge by potentially providing safe support to perform more challenging and intense therapy during acute inpatient rehabilitation in TBI. This retrospective analysis suggests therapy sessions using DBWS can be achieved by a single therapist. Our findings also suggest that DBWS leads to greater improvement in total FIM compared to SOC. It is established

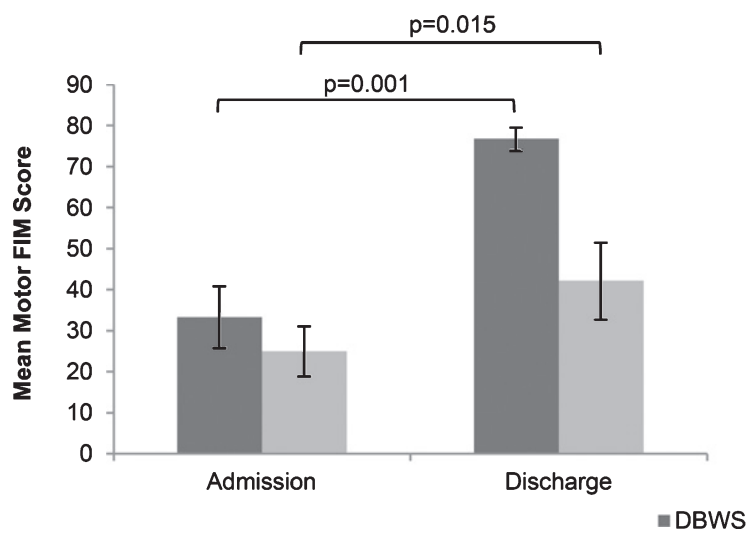

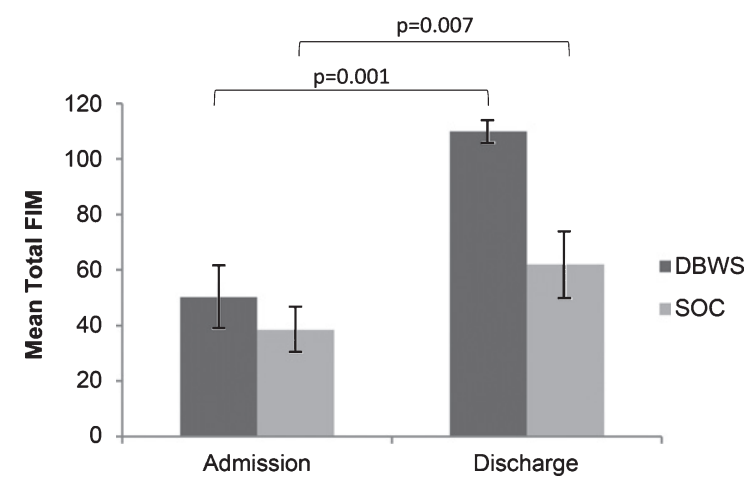

Fig. 1. The mean total FIM score on admission and discharge was greater for patients using the Zero $\mathrm{G}$ versus SOC.

that intensive therapy leads to neuroplastic changes resulting in better functional improvements. However, there are barriers to applying intensive therapy, particularly in a very impaired population in an inpatient setting. Most of the studies which use concepts of neuroplasticity such as intensive therapy have been in stroke populations and very few in TBI. Most studies in TBI have applied DBWS and treadmill therapy

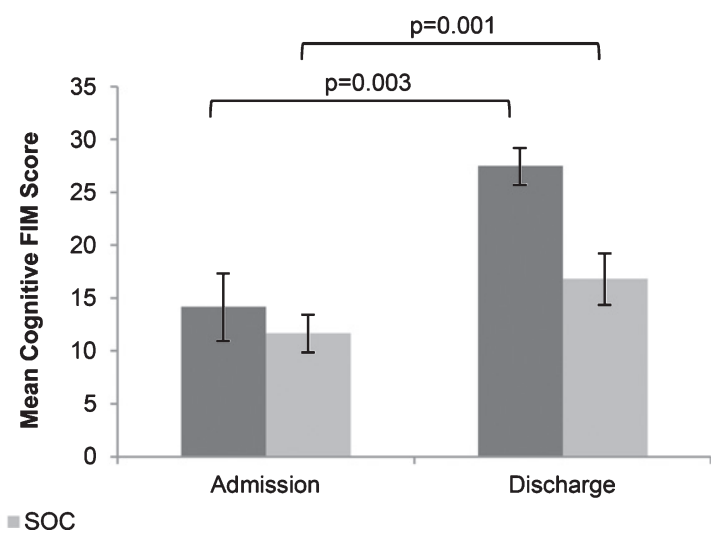

Fig. 2. The mean Motor FIM and Cognitive FIM subscales on admission and discharge were greater for patients using the Zero G versus SOC. 


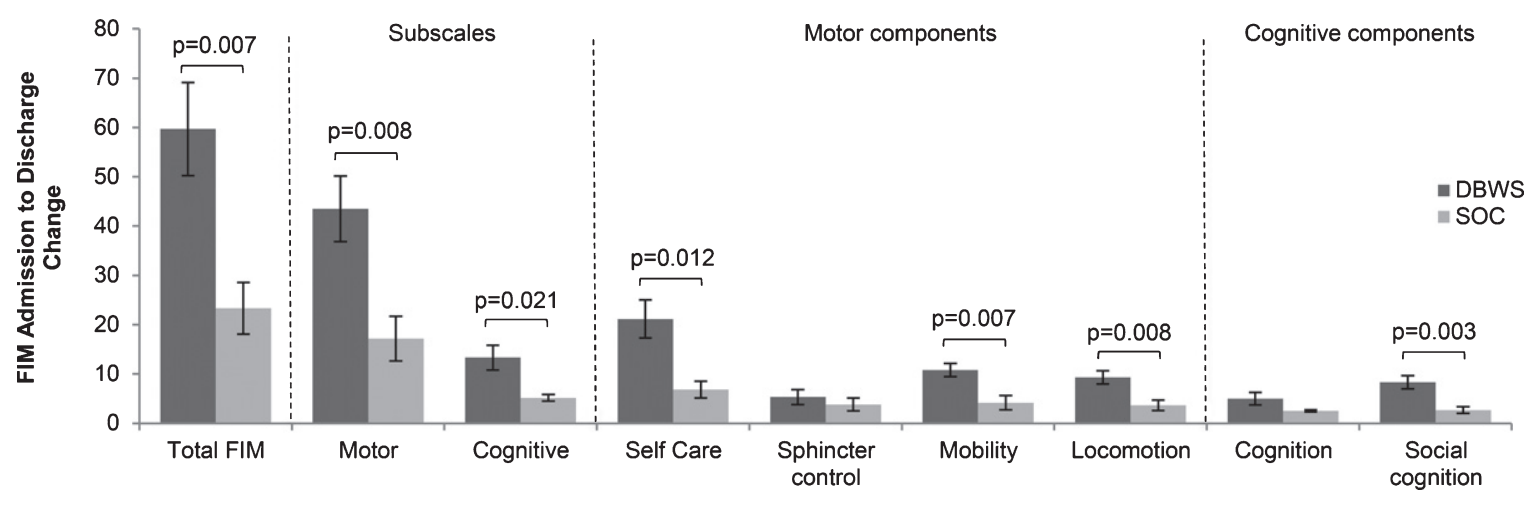

Fig. 3. The differences in mean FIM score on admission to discharge showed statistical significance in both cognitive and motor function in patients using the DBWS versus SOC.

with minimal to no beneficial effects (Bland et al., 2011; Brown et al., 2005). One study in 22 patients with chronic TBI compared 3 types of locomotor therapy equipment to determine if any of them promoted better outcomes (Esquenazi et al., 2017). No evidence of significant difference was found among the 3 devices, but the Lokomat did require fewer therapists. Brown et al. evaluated the effects of treadmill therapy compared to conventional over-ground gait therapy in 20 participants more than 6 years postinjury. There was improvement in gait symmetry only in the conventional gait therapy group (Brown et al., 2005). However, it is difficult to perform overground therapy due to the required number of staff for patient safety. Therefore, clinicians need methods for applying intensive therapy without requiring multiple therapists, particularly in an acute inpatient setting. Due to staffing limitations, patients do not receive therapy at the optimal intensity and/or repetitions, especially in those with both motor and cognitive impairments. Our study shows that the use of DBWS allowed the therapists to administer more intense therapy, challenge patients and encourage them to attempt movement, while simultaneously relieving them of their fear of falling.

Our results also suggest that both cognitive and motor impairments can be decreased in a TBI population. While the mechanism underlying this improvement needs to be better understood, it is conceivable that cognitive improvement is due to the patient's upright positioning thus providing more stimulation to the brainstem and/or increased blood flow to the brain. Studies have shown that aerobic exercise improves cognitive performance and the upright position increases arousal, decreases parasympathetic activity, and improvement in problem solving and stimuli detection tasks (Jonasson et al., 2016; Muehlhan, Marxen, Landsiedel, Malberg, \& Zaunseder, 2014; Smith et al., 2010). Additionally, it is possible that because the patient is not afraid of falling, he/she is able to pay more attention to the task which may also help to improve cognitive function. Most interventions in TBI have targeted cognitive OR motor impairment, and devices such as DBWS may be a novel way to target both domains.

\subsection{Study limitations}

Limitations of this study include having a small sample size and the retrospective nature of the study. Additionally, this study offered no way to monitor for intensity of the therapy, and the activities were heterogeneous from patient to patient and session to session. However, to our knowledge, this is the first study to attempt to evaluate the effectiveness of these devices in an inpatient study. Future studies will need a larger sample size, be better controlled, and have longer term follow-ups. Additionally, more specific outcome measures for cognition including attention, memory, and executive function, and more specific outcome measures for motor including muscle weakness, spasticity, balance, and coordination disturbances. Furthermore, future studies should also evaluate if therapies incorporating DBWS during over-ground therapy can lead to increased community reintegration after TBI. Follow-ups will be important to determining community re-integration.

\section{Conclusion}

This feasibility study shows promising data that DBWS may provide beneficial effects to TBI in both 
cognitive and motor components. It allows therapists to provide intensive therapy following the principles of neuroplasticity in a safe manner. Our promising data warrants larger prospective randomized controlled studies.

\section{Conflict of interest}

This study was funded by the Cardinal Hill Rehabilitation Hospital Endowed Chair in Stroke and Spinal Cord Injury Rehabilitation (0705129700) and supported by the HealthSouth Therapy Grant. The funding body had no role in the collection, analysis and interpretation of data, or in writing the manuscript. There are no financial benefits to the authors. There are no conflicts of interest related to this research or this manuscript.

\section{References}

Arciniegas, D. B., Held, K., \& Wagner, P. (2002). Cognitive Impairment Following Traumatic Brain Injury. Curr Treat Options Neurol, 4(1), 43-57.

Beaulieu, C. L., Peng, J., Hade, E. M., Corrigan, J. D., Seel, R. T., Dijkers, M. P., \& Bogner, J. (2019). Impact of Level of Effort on the Effects of Compliance with the 3-Hour Rule. Arch Phys Med Rehabil. doi:10.1016/j.apmr.2019.01.014

Bland, D. C., Zampieri, C., \& Damiano, D. L. (2011). Effectiveness of physical therapy for improving gait and balance in individuals with traumatic brain injury: a systematic review. Brain Inj, 25(7-8), 664-679. doi:10.3109/02699052.2011.576306

Brasure, M., Lamberty, G. J., Sayer, N. A., Nelson, N. W., MacDonald, R., Ouellette, J., \& Wilt, T. J. (2012) Multidisciplinary Postacute Rehabilitation for Moderate to Severe Traumatic Brain Injury in Adults. Rockville (MD).

Brown, T. H., Mount, J., Rouland, B. L., Kautz, K. A., Barnes, R. M., \& Kim, J. (2005). Body weight-supported treadmill training versus conventional gait training for people with chronic traumatic brain injury. The Journal of head trauma rehabilitation, 20(5), 402-415.

Centers for Disease Control and Prevention. Traumatic Brain Injury \& Concussion. (April 27, 2017). https://www.cdc.gov/ traumaticbraininjury/get_the_facts.html.

Corso, P., Finkelstein, E., Miller, T., Fiebelkorn, I., \& Zaloshnja, E. (2006). Incidence and lifetime costs of injuries in the United States. Inj Prev, 12(4), 212-218. doi:10.1136/ip.2005.010983

Dobkin, B. H. (1993). Neuroplasticity. Key to recovery after central nervous system injury. West J Med, 159(1), 56-60.
Esquenazi, A., Lee, S., Wikoff, A., Packel, A., Toczylowski, T., \& Feeley, J. (2017). A Comparison of Locomotor Therapy Interventions: Partial-Body Weight-Supported Treadmill, Lokomat, and G-EO Training in People With Traumatic Brain Injury. PM $R, 9(9), 839-846$. doi:10.1016/j.pmrj.2016.12.010

Glees, P., \& Cole, J. (1949). The reappearance of coordinated movements of the hand after lesions in the hand area of the motor cortex of the rhesus monkey. J Physiol, 108(1), Proc, 33.

Jonasson, L. S., Nyberg, L., Kramer, A. F., Lundquist, A., Riklund, K., \& Boraxbekk, C. J. (2016). Aerobic Exercise Intervention, Cognitive Performance, and Brain Structure: Results from the Physical Influences on Brain in Aging (PHIBRA) Study. Front Aging Neurosci, 8, 336. doi:10.3389/fnagi.2016.00336

Kleim, J. A., \& Jones, T. A. (2008). Principles of experiencedependent neural plasticity: implications for rehabilitation after brain damage. J Speech Lang Hear Res, 51(1), S225-239. doi:51/1/S225 [pii] 10.1044/1092-4388(2008/018)

Mao, Y. R., Lo, W. L., Lin, Q., Li, L., Xiao, X., Raghavan, P., \& Huang, D. F. (2015). The Effect of Body Weight Support Treadmill Training on Gait Recovery, Proximal Lower Limb Motor Pattern, and Balance in Patients with Subacute Stroke. BioMed research international, 2015, 175719. doi: $10.1155 / 2015 / 175719$

Muehlhan, M., Marxen, M., Landsiedel, J., Malberg, H., \& Zaunseder, S. (2014). The effect of body posture on cognitive performance: a question of sleep quality. Frontiers in human neuroscience, 8, 171. doi:10.3389/fnhum.2014.00171

Nudo, R. J. (2011). Neural bases of recovery after brain injury. $J$ Commun Disord, 44(5), 515-520. doi:S00219924(11)00026-8 [pii] 10.1016/j.jcomdis.2011.04.004

Peterson, M., \& Greenwald, B. D. (2015). Balance problems after traumatic brain injury. Arch Phys Med Rehabil, 96(2), 379-380. doi:10.1016/j.apmr.2013.06.012

Smith, P. J., Blumenthal, J. A., Hoffman, B. M., Cooper, H., Strauman, T. A., Welsh-Bohmer, K., \& Sherwood, A. (2010). Aerobic exercise and neurocognitive performance: a metaanalytic review of randomized controlled trials. Psychosom Med, 72(3), 239-252. doi:10.1097/PSY.0b013e3181d14633

Turner-Stokes, L., Pick, A., Nair, A., Disler, P. B., \& Wade, D. T. (2015). Multi-disciplinary rehabilitation for acquired brain injury in adults of working age. Cochrane Database Syst $\operatorname{Rev}(12)$, CD004170. doi:10.1002/14651858.CD004170.pub3

Weaver, J. (2015). Motor Learning Unfolds over Different Timescales in Distinct Neural Systems. PLoS Biol, 13(12), e1002313. doi:10.1371/journal.pbio.1002313

Wolf, S. L., Winstein, C. J., Miller, J. P., Taub, E., Uswatte, G., Morris, D., \& Nichols-Larsen, D. (2006). Effect of constraintinduced movement therapy on upper extremity function 3 to 9 months after stroke: the EXCITE randomized clinical trial. JAMA, 296(17), 2095-2104. doi:10.1001/jama.296.17.2095 